\title{
Clinical Results of Radiotherapy for Locally Advanced Stage III Pan- creatic Cancer: A Single Institutional Experience
}

\author{
Yoshiomi Hatayama ${ }^{1}$, Masahiko Aoki ${ }^{1}$, Hideo Kawaguchi ${ }^{1}$, Katsumi Hirose ${ }^{2}$, Mariko \\ Sato $^{1}$, Ichitaro Fujioka ${ }^{1}$, Mitsuki Tanaka ${ }^{1}$, Koji Ichise ${ }^{1}$, Shuichi Ono ${ }^{1}$ and Yoshihiro Takai ${ }^{2}$ \\ ${ }^{1}$ Department of Radiology and Radiation Oncology, Hirosaki University Graduate School of Medicine, Japan \\ ${ }^{2}$ Southern Tohoku BNCT Research Center, Fukushima, Japan
}

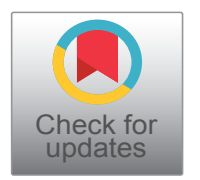

*Corresponding author: Yoshiomi Hatayama, Department of Radiology and Radiation Oncology, Hirosaki University Graduate School of Medicine, 5 Zaifu-cho, Hirosaki, Aomori 036-8562, Japan, Tel: +81-172-39-5103, Fax: +81-172-335627, E-mail: yhatayama@hirosaki-u.ac.jp

\begin{abstract}
Objectives: We conducted an evaluation of the efficacy and toxicity of radiotherapy for locally advanced stage III pancreatic cancer.

Methods: Fifteen patients with locally advanced stage III pancreatic cancer underwent radiotherapy with or without concurrent chemotherapy between July 2006 and April 2014. We used 10 MV X-rays and multiple coplanar (two to four) fields. The number of fractions ranged between 20 and 28 with a fraction size of $1.8 \mathrm{~Gy}$. A total dose of $36-50.4 \mathrm{~Gy}$ at the isocenter of the planning target volume (PTV) was administered to each patient. Fourteen patients received chemotherapy during radiotherapy. The cumulative survival rate and local control rate were calculated using the Kaplan-Meier method.
\end{abstract}

Results: The study included nine males and six females with a median age of 61 years (age range: $42-85$ years). The tumor stage was T4 in all patients and lymph node metastasis was $\mathrm{N} 1$ in 11 patients and N0 in 4. The clinical stage in the UICC $7^{\text {th }}$ was III in all patients. The median follow-up period was 7.8 months. For a total of 15 patients, the oneand two-year overall survival rates were $38.9 \%$ and $12.9 \%$, respectively. The six-month and one-year local progression-free survival rates were $59.4 \%$ and $0 \%$, respectively.

Conclusion: We reported the clinical outcomes of locally advanced stage III pancreatic cancer in a single institution. Although this treatment option is a feasible, the efficacy should remain to be verified with future large-scale studies.

\section{Keywords}

Locally advanced pancreatic cancer, Radiotherapy, Chemotherapy

\section{Introduction and Objectives}

In Japan, pancreatic cancer represents the fourth leading cause of death due to cancer [1]. Recently, diagnostic methods and treatment technology for pancreatic cancer has been progressed; however, the mortality rate has not declined. At the time of the initial diagnosis, there are few patients who are resectable absence of the invasion of major vessels or the presence of distant metastasis. The three-year overall survival rate of the patients who were resectable is reported to be only $20 \%$ [2]. On the other hand, according to a report from approximately 10-years-ago, the median survival time of the patients who were unresectable was approximately between 6 and 13 months [3-8]. Thus, regardless of the enforcement of surgery, the prognosis of pancreatic cancer is extremely poor compared to other cancers. For patients with unresectable pancreatic cancer, the benefit of the addition of chemotherapy to radiotherapy has proven $[8,9]$; however, the effect of the addition of radiotherapy to chemotherapy is unclear [10]. Regarding radiotherapy, due to the poor prognosis and insufficient cases of pancreatic cancer, high-quality evidences concerning the treatment strategy are scarce. 5FU combination radiotherapy has become one of the standard treatments, but the relevant supporting literature is dated. The purpose of this study is to retrospectively evaluate the efficacy and toxicity of radiotherapy for locally advanced stage III pancreatic cancer in our institution. 


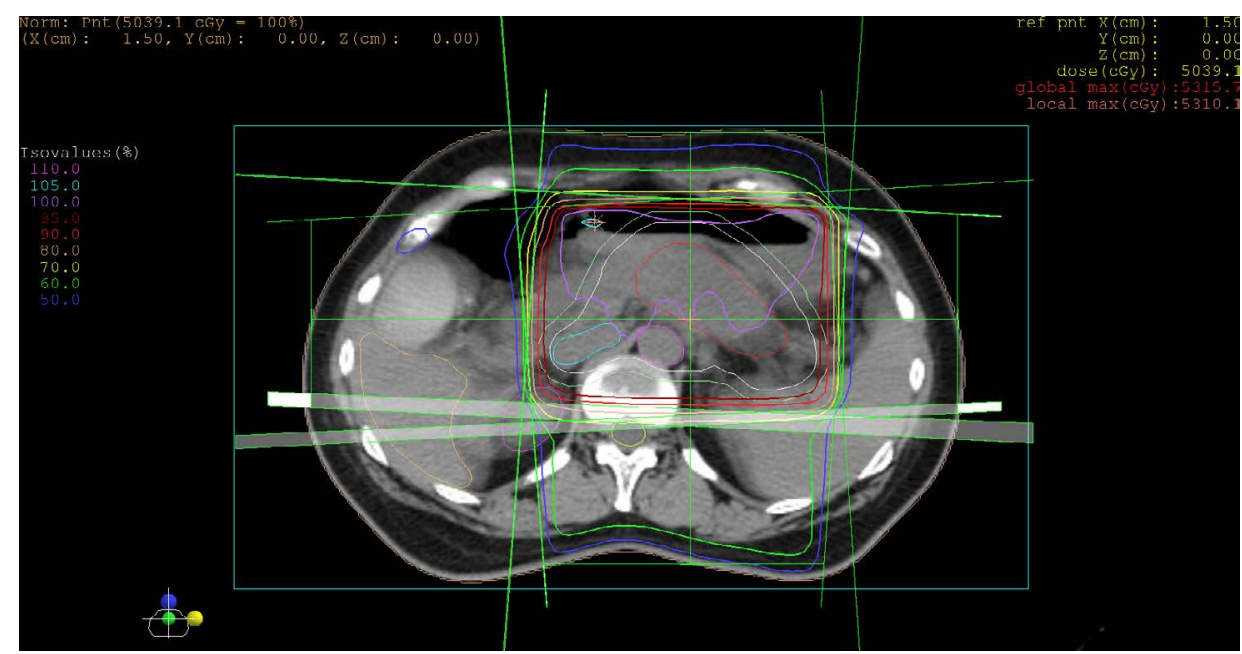

Figure 1: Three-dimensional dose distributions for unresectable stage III pancreatic cancer that received a total dose of 50.4 Gy using 10 MV X-rays and four coplanar fields.

\section{Methods and Materials}

\section{Patients}

We retrospectively investigated 15 patients with locally advanced stage III pancreatic cancer who underwent radiotherapy with or without concurrent chemotherapy at our institution between July 2006 and April 2014. Written informed consent was provided from all patients following an explanation of the clinical stage and life prognosis, treatment goals, treatment schedule, other treatment options, and adverse events. The complete patient evaluation included a physical examination, blood counts, screening blood chemistry tests, and an electrocardiogram. The clinical TNM staging (UICC) was performed using chest and abdominal radiographs, chest-abdominal computed tomography (CT), and/or positron emission tomography (PET/CT) scans. This study was approved by the institutional review board of our institution.

\section{Radiotherapy}

We used a three-dimensional (3D) radiotherapy planning procedure with high-energy linear accelerators. Serial CT scans with $2.5-\mathrm{mm}$ intervals were performed. Following the CT scan, the organs at risk (i.e., duodenum, liver, bilateral kidney and spinal cord) and target outlines were drawn. The gross tumor volume (GTV) was defined as primary tumor and enlarged lymph nodes detected by CT scans and/or PET-CT scans. The irradiated clinical target volume (CTV) included the GTV and regional lymph nodes area (i.e., pancreaticoduodenal and celiac axis). The planning target volume (PTV) was defined by the CTV plus $1-1.5 \mathrm{~cm}$ margins for movement and uncertainties during the set up. We used 10 MV X-rays and multiple coplanar (two to four) fields. The number of fractions ranged between 20 and 28 in fractions of $1.8 \mathrm{~Gy} /$ day, five days per week. A total dose of 36-50.4Gy at the isocenter of the PTV was administered to each patient. One representative case of the 3D radiotherapy planning for locally advanced pancreatic body cancer is shown in (Figure 1). The dose constraints of the organs at risk were evaluated by a dose volume histogram. The restriction for the liver, kidney, and spinal cord were $\leq 30 \mathrm{~Gy}, \leq 20 \mathrm{~Gy}$, and $\leq 40 \mathrm{~Gy}$, respectively.

\section{Evaluation and analysis}

The objectives of this study were to evaluate the local control, overall survival, and toxicity. The patients were monitored for a follow-up every two to three months during the first year, and every four to six months thereafter. The majority of the patients were followed by clinical examination, CT scans, and laboratory data, including tumor markers. The local tumor response was evaluated using CT scan and the Response Evaluation Criteria in Solid Tumors (RECIST) [11]. Local tumor control was defined as a lack of any significant tumor regrowth on the follow-up CT. The cumulative survival rates and local control rates from the first date of treatment until the date of death or local recurrence were calculated using the Kaplan-Meier method. These were estimated from the date of the radiotherapy initiation to the date of event or the last follow-up. All analyses were performed using Prism v5.0f (GraphPad Software, Inc., USA). The grade of treatment toxicity was evaluated using the Common Terminology Criteria for Adverse Events (CTCAE) v3.0. Acute and subacute toxicities were defined as occurring within six months after the radiotherapy, and late toxicities were defined at six months or later.

\section{Results}

\section{Patient characteristics}

A summary of the patients' characteristics is provided in Table 1. The nine men and six women who comprised the cohort had a median age of 61-years (range: 42-85 years). At the time of the analysis, eight patients had died, and seven patients were alive. The median follows up was 7.8 months (range: 1.2-18.8 months) for all patients and 4.1 months (range: 1.2-18.8 months) for 
(a)

Overall survival rate

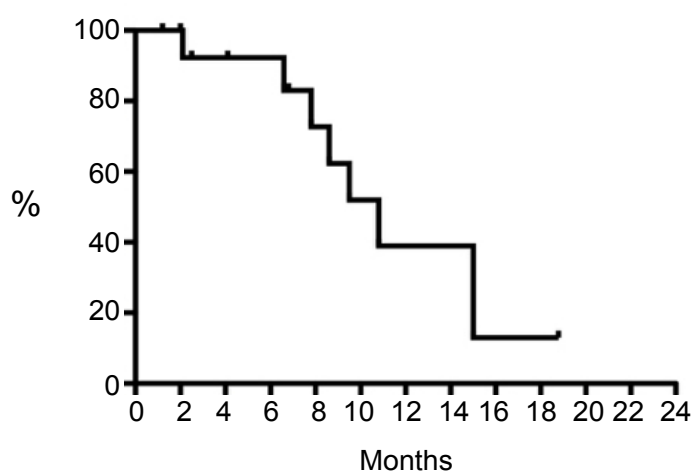

(b)

Local control rate

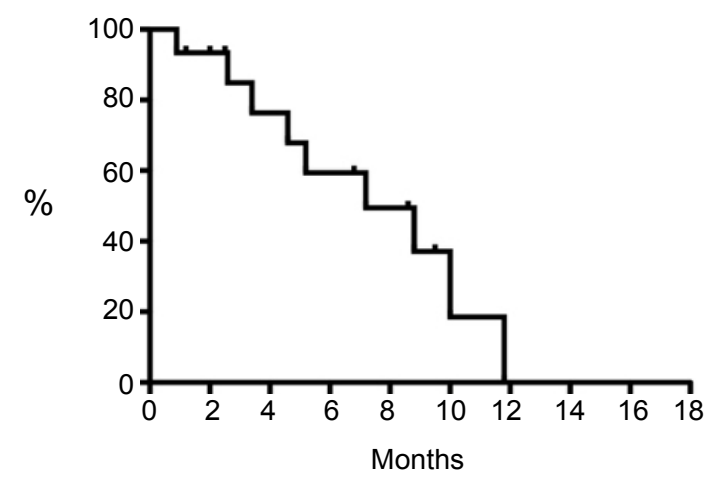

Figure 2: (a) Overall survival rate for 15 patients with unresectable stage III pancreatic cancer treated with radiotherapy; (b) Local control rate for 15 patients with unresectable stage III pancreatic cancer treated with radiotherapy.

Table 1: Patient Characteristics $(n=15)$.

\begin{tabular}{|c|c|c|}
\hline & Characteristic & Value \\
\hline \multicolumn{3}{|l|}{ Sex } \\
\hline & Male & $9(60 \%)$ \\
\hline & Female & $6(40 \%)$ \\
\hline \multicolumn{3}{|l|}{ Age } \\
\hline & Median (range) & $61(42-85)$ \\
\hline \multicolumn{3}{|l|}{ Performance Status } \\
\hline & $0-1$ & $13(87 \%)$ \\
\hline & 2 & $2(13 \%)$ \\
\hline \multicolumn{3}{|l|}{ Stage } \\
\hline & cT4N0 & $4(27 \%)$ \\
\hline & cT4N1 & $11(73 \%)$ \\
\hline \multicolumn{3}{|l|}{ Site of pancreas } \\
\hline & Head & $8(53.3 \%)$ \\
\hline & Body & $5(33.3 \%)$ \\
\hline & Tail & $2(13.3 \%)$ \\
\hline \multicolumn{3}{|l|}{ Radiation doses (Gy) } \\
\hline & Median (range) & 50.4Gy (36-50.4Gy) \\
\hline \multicolumn{3}{|l|}{$\begin{array}{l}\text { Combination of } \\
\text { Chemotherapy }\end{array}$} \\
\hline & Yes & $14(93 \%)$ \\
\hline & No & $1(7 \%)$ \\
\hline \multicolumn{3}{|l|}{$\begin{array}{l}\text { Follow-up Time } \\
\text { (month) }\end{array}$} \\
\hline & Median (range) & $7.8(1.2-18.8)$ \\
\hline \multicolumn{3}{|c|}{ 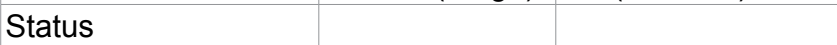 } \\
\hline & Alive & $7(47 \%)$ \\
\hline & Dead & $8(53 \%)$ \\
\hline \multicolumn{3}{|l|}{ Failure } \\
\hline & Yes & $12(80 \%)$ \\
\hline & No & $3(20 \%)$ \\
\hline
\end{tabular}

the surviving patients. Four patients were lost to follow-up. The performance status was between 0 and 1 in 13 patients and 2 in 2 patients. The tumor stage was T4 in all patients. Unresectable and borderline resectable were 11 patients and 4 patients, respectively. The lymph node metastasis was N1 in 11 patients, and NO in 4 . The clinical stage in the UICC $8^{\text {th }}$ was III for all patients. The histological types were adenocarcinoma in 7 patients
Table 2: Failure Patterns after radiotherapy.

\begin{tabular}{|l|l|}
\hline Sites of Failures & Number of Patients \\
\hline Locoregional & 9 \\
\hline Distant metastasis & 8 \\
\hline Liver & 6 \\
\hline Pulmonary & 1 \\
\hline Hilar lymph node & 1 \\
\hline
\end{tabular}

(47\%); 8 tumors could not be diagnosed by biopsy or cytology and had been clinically diagnosed. Clinical diagnosis of malignancy was based on CT scan, or uptake on PET/CT scan. The median dose of radiotherapy was $50.4 \mathrm{~Gy}$. The 3D radiotherapy used four portals in $12 \mathrm{pa}-$ tients, three portals in 2 patients, and two portals in 1. Fourteen patients received chemotherapy during radiotherapy. The concurrent chemotherapy was TS-1 in 12 patients, gemcitabine (GEM) and TS-1 in 1 patient, and GEM in 1 patient. The GEM-based regimen had been administered to nine patients before radiotherapy as an initial treatment. The duration of prior chemotherapy was 3 to 6 months.

\section{Survival and local tumor control}

For a total 15 patients, the one- and two-year overall survival rates were $38.9 \%$ and $12.9 \%$, respectively, and the six-month and one-year local progression-free survival rates were $59.4 \%$ and $0 \%$, respectively (Figure 2 ). The site of the initial failure among the 15 patients is provided in Table 2. During the follow-up, local progression occurred in nine patients (60\%). Eight patients (53.3\%) developed distant metastasis, six developed liver metastasis, one developed pulmonary metastasis, and one developed hilar lymph node metastasis. Two patients developed local progression and distant metastasis simultaneously. In all eight patients who had died, death was a result of disease progression.

\section{Toxicities}

Leukopenia and thrombocytopenia of CTCAE criteria 
Table 3: Previous reports of chemoradiotherapy with TS-1 for locally advanced pancreatic cancer.

\begin{tabular}{|l|l|l|l|l|}
\hline Author & Number of Patients & Radiotherapy & Median Survival time & $1 \mathrm{y}$-OS \\
\hline Ikeda 2007 & 21 & $50.4 G y$ & 11 & $42.90 \%$ \\
\hline Kim 2009 & 25 & $50.4 G y$ & 12.9 & $43 \%$ \\
\hline Sudo 2011 & 34 & $50.4 G y$ & 16.8 & $70.60 \%$ \\
\hline Shinchi 2012 & 50 & $50 G y$ & 14.3 & $62 \%$ \\
\hline Our study & $12 / 15$ & $36-50.4 G y$ & 9 & $38.70 \%$ \\
\hline
\end{tabular}

Abbreviations: 1y-OS, 1-year overall survival rate.

Grade 2 was observed in three (20\%) and one (6.7\%) patient, respectively. No other severe adverse events $(\geq$ grade 3 ) have been observed in any of the patients as of the last follow-up.

\section{Discussion}

Chauffert, et al. conducted a randomized controlled trial to evaluate the use of chemoradiotherapy for locally advanced pancreatic cancer with Fluorouracil and Cisplatin and chemotherapy alone with Gemcitabine; the authors reported that the survival time of the chemotherapy alone group was significantly better than chemoradiotherapy group in 2008 [12]. Furthermore, the incidence of severe adverse events was significantly higher in the chemoradiotherapy group. In contrast, Loehrer, et al. conducted a randomized controlled trial to investigate the use of chemoradiotherapy with Gemcitabine and chemotherapy alone with Gemcitabine. They reported that the survival time of the chemoradiotherapy group was significantly longer than the chemotherapy alone group in 2011 [13]. Regarding adverse events, the incidence of grade 3-4 did not differ between the two groups. As described above, it is not possible conclude the superiority of either chemoradiotherapy or chemotherapy alone at this time. Therefore, currently the first line treatment for locally advanced pancreatic cancer has recommended the administration of chemoradiotherapy or chemotherapy alone. In our experience, chemotherapy alone has been widespread in clinical practice and there are less opportunities to implement radiotherapy with or without chemotherapy for locally advanced pancreatic cancer. This study included only 15 patients in eight years however with such a small sample size, the number of patients that can receive radiotherapy are minimal, and similar published reports also involve small sample sizes.

From the 15 patients included in this study, the one-year overall survival rate and median survival time were $38.9 \%$ and 10.8 months, respectively. The median survival time of the patients with locally advanced pancreatic cancers that received chemoradiotherapy or radiotherapy alone or chemotherapy alone was found to be between 5.7-16.8 in a previous randomized controlled study [8-10,12-16]. In particular, the results of the median survival time with chemoradiotherapy with TS-1 had been 11-16.8 months [17-22]. Our study included 12 patients with TS- 1 and a median survival time of 10.8 months, which is slightly inferior compared to previous reports. We summarized previous reports of the chemoradiotherapy with TS- 1 for locally advanced pancreatic cancer in Table 3.

Unfortunately, both the one-year local control rate and progression free survival rate in our study were $0 \%$, respectively. Local disease recurrence and distant metastasis occurred in nine $(60 \%)$ and eight patients (53.3\%), respectively. Moreover, both local disease recurrence and distant metastasis occurred in five patients (33.3\%). Only three (20\%) patients have experienced a period without local disease recurrence and distant metastasis. The most frequent site of distant metastasis was the liver in six patients (40\%). The venous drainage from the pancreas flows to the liver via the portal vein. Therefore, distant metastasis of pancreatic cancer is most often liver metastasis, and our results are in line with this observation. A total of $9(60 \%)$ out of 15 patients received chemotherapy before chemoradiotherapy and $8(66.7 \%)$ out of 12 patients with local disease recurrence or distant metastasis underwent chemotherapy with gemcitabine prior to chemoradiotherapy with TS-1. Many of these patients were consulted regarding radiotherapy because chemotherapy was not effective in our institution. Thus, patients who underwent chemoradiotherapy as an initial treatment was less and chemoradiotherapy was performed under severe conditions. This is considered to be one of the reasons why the clinical outcomes of this study were slightly inferior as compared to previous reports.

Regarding to adverse events, three patients developed grade 2 leukopenia and one patient developed grade 2 thrombopenia. Six patients developed grade 1 gastrointestinal adverse events such as anorexia and nausea. No grade 3 or greater toxicities were observed in this study. Therefore, the results of this study indicate that radiotherapy with TS-1 and/or GEM is a feasible and relatively safe treatment option in locally advanced pancreatic cancer. As of the last follow-up, no severe late adverse events occurred in any of our patients.

In this study, we reported the clinical outcomes of locally advanced stage III pancreatic cancer at a single institution. Although this treatment option is feasible, the efficacy is not satisfactory. In addition, we have obtained similar results as other reports, with the exception of the particle beam therapy. With the appearance of some new anti-cancer agents, the survival period has been extended $[23,24]$. However, compared to other cancers, the therapeutic effect remains poor. In the future, by combining radiotherapy and chemotherapy, we 
hope that the clinical outcomes of locally advanced pancreatic cancer will be improved.

\section{Conflict of Interest Disclosures}

We have read and understood International Journal of Oncology Research's policy on disclosing conflicts of interest, and we declare that we have none.

\section{References}

1. (2016) Latest Cancer Statistics. National Cancer Research Center, Cancer Control and Information Center, National Research and Development Institute, Japan.

2. Doi R, Imamura M, Hosotani R, Imaizumi T, Hatori T, et al (2008) Surgery versus radiochemotherapy for resectable locally invasive pancreatic cancer: Final results of a randomized multi-institutional trial. Surg Today 38: 1021-1028.

3. Ishii H, Okada S, Tokuuye K, Nose H, Okusaka T, et al. (1997) Protracted 5-fluorouracil infusion with concurrent radiotherapy as a treatment for locally advanced pancreatic carcinoma. Cancer 79: 1516-1520.

4. Ueno H, Okusaka T, Ikeda M, Tokuuye K (2004) Phase I study of hyperfractionated radiation therapy with protracted 5-fluorouracil infusion in patients with locally advanced pancreatic cancer. Oncology 67: 215-221.

5. Rich T, Harris J, Abrams R, Erickson B, Doherty M, et al. (2004) Phase II study of external irradiation and weekly paclitaxel for nonmetastatic, unresectable pancreatic cancer: RTOG-98-12. Am J Clin Oncol 27: 51-56.

6. Huguet F, André T, Artru P, Balosso J, Selle F, et al. (2007) Impact of chemoradiotherapy after disease control with chemotherapy in locally advanced pancreatic adenocarcinoma in GERCOR phase II and III studies. J Clin Oncol 25: 326-331.

7. Wilkowski R, Thoma M, Bruns C, Wagner A, Heinemann V, et al. (2006) Chemoradiotherapy with gemcitabine and continuous 5-FU in patients with primary inoperable pancreatic cancer. JOP 7: 349-360.

8. Moertel CG, Frytak S, Hahn RG, O'Connell MJ, Reitemeier RJ, et al. (1981) Therapy of locally unresectable pancreatic carcinoma: A randomized comparison of high dose $(6000$ rads) radiation alone, moderate dose radiation (4000 rads + 5-fluorouracil), and high dose radiation +5 -fluorouracil: The Gastrointestinal Tumor Study Group. Cancer 48: 1705-1710.

9. Moertel CG, Childs DS Jr, Reitemeier RJ, Colby MY Jr, Holbrook MA, et al. (1969) Combined 5-fluorouracil and supervoltage radiation therapy of locally unresectable gastrointestinal cancer. Lancet 2: 865-867.

10. Klaassen DJ, Maclntyre JM, Catton GE, Engstrom PF, Moertel CG, et al. (1985) Treatment of locally unresectable cancer of the stomach and pancreas: A randomized comparison of 5-fluorouracil alone with radiation plus concurrent and maintenance 5-fluorouracil--an Eastern Cooperative Oncology Group study. J Clin Oncol 3: 373-378.

11. Eisenhauer EA, Therasse P, Bogaerts J, Schwartz LH, Sargent D, et al. (2009) New response evaluation criteria in solid tumours: Revised RECIST guideline (version 1.1). Eur J Cancer 45: 228-247.

12. Chauffert B, Mornex F, Bonnetain F, Rougier $P$, Mariette $C$, et al. (2008) Phase III trial comparing intensive induction chemoradiotherapy (60 Gy, infusional 5-FU and intermittent cisplatin) followed by maintenance gemcitabine with gemcitabine alone for locally advanced unresectable pancre- atic cancer. Definitive results of the 2000-01 FFCD/SFRO study. Ann Oncol 19: 1592-1599.

13. Loehrer PJ Sr, Feng Y, Cardenes H, Lynne Wagner, Joanna M Brell, et al. (2011) Gemcitabine alone versus gemcitabine plus radiotherapy in patients with locally advanced pancreatic cancer: An Eastern Cooperative Oncology Group trial. J Clin Oncol 29: 4105-4112.

14. Cohen SJ, Dobelbower R Jr, Lipsitz S, Catalano PJ, Sischy $B$, et al. (2005) A randomized phase III study of radiotherapy alone or with 5-fluorouracil and mitomycin-C in patients with locally advanced adenocarcinoma of the pancreas: Eastern Cooperative Oncology Group study E8282. Int J Radiat Oncol Biol Phys 62: 1345-1350.

15. Shinchi H, Takao S, Noma H, Matsuo $Y$, Mataki $Y$, et al. (2002) Length and quality of survival after external-beam radiotherapy with concurrent continuous 5-fluorouracil infusion for locally unresectable pancreatic cancer. Int J Radiat Oncol Biol Phys 53: 146-150.

16. Li CP, Chao Y, Chi KH, Chan WK, Teng HC, et al. (2003) Concurrent chemoradiotherapy treatment of locally advanced pancreatic cancer: Gemcitabine versus 5-fluorouracil, a randomized controlled study. Int J Radiat Oncol Biol Phys 57: 98-104.

17. Sudo K, Yamaguchi T, Ishihara T, Kazuyoshi Nakamura, Yoshihiko Shirai, et al. (2007) Phase I study of oral S- ${ }_{1}$ and concurrent radiotherapy in patients with unresectable locally advanced pancreatic cancer. Int J Radiat Oncol Biol Phys 67: 219-224.

18. Ikeda M, Okusaka T, Ito Y, H Ueno, C Morizan, et al. (2007) A phase I trial of S-1 with concurrent radiotherapy for locally advanced pancreatic cancer. Br J Cancer 96: 1650-1655.

19. Kim HM, Bang S, Park JY, Jinsil Seong, Si Young Song, et al. (2009) Phase II trial and concurrent radiotherapy in patients with locally advanced pancreatic cancer. Cancer Chemother Pharmacol 63: 535-541.

20. Sudo K, Yamaguchi T, Ishihara T, Nakamura K, Hara T, et al. (2011) Phase II study of oral S-1 and concurrent radiotherapy in patients with unresectable locally advanced pancreatic cancer. Int J Radiat Oncol Biol Phys 80: 119-125.

21. Shinchi H, Maemura K, Mataki Y, Hiroshi Kurahara, Masahiko Sakoda, et al. (2012) A phase II study of oral S-1 with radiotherapy followed by chemotherapy with S-1 alone for locally advanced pancreatic cancer. J Hepatobiliary Pancreat Sci 19: 152-158.

22. Shinchi H, Maemura K, Noma H, Y Mataki, T Aikou, et al. (2007) Phase-I trial of oral fluoropyrimidine anticancer agent (S-1) with concurrent radiotherapy in patients with unresectable pancreatic cancer. Br J Cancer 96: 1353-1357.

23. Yamazaki H, Nishiyama K, Koizumi M, Tanaka E, loka T, et al. (2007) Concurrent chemoradiotherapy for advanced pancreatic cancer: $1,000 \mathrm{mg} / \mathrm{m} 2$ gemcitabine can be administered using limited-field radiotherapy. Strahlenther Onkol 183: 301-306.

24. Shibuya K, Oya N, Fujii T, Doi R, Nakamura A, et al. (2011) Phase II study of radiation therapy combined with weekly low-dose gemcitabine for locally advanced, unresectable pancreatic cancer. Am J Clin Oncol 34: 115-119.
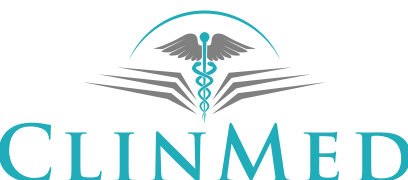

INTERNATIONAL LIBRARY 